\title{
Saint Bavon et les origines de Gand
}

\section{Adriaan Verhulst}

\section{Citer ce document / Cite this document :}

Verhulst Adriaan. Saint Bavon et les origines de Gand. In: Revue du Nord, tome 68, n²69, Avril-juin 1986 . Saint Géry et la christianisation dans le nord de la Gaule Ve-IXe siècles. Actes du colloque de Cambrai 5-7 octobre 1984. pp. 455-470; doi : https://doi.org/10.3406/rnord.1986.4226

https://www.persee.fr/doc/rnord_0035-2624_1986_num_68_269_4226

Fichier pdf généré le 08/04/2018 
Deze studie wil in de eerste plaats bepalen waar de broeders van een kort daarvoor door Sint Amands aan de samenvloeing van Leie en Schelde, te Gent, gestichte gemeenschap een eel bouwden voor kluizenaar Bavo. De auteur situeert deze eel op de plaats van de huidige Sint-Baafskathedraal, waar, volgens hem, voor 804 de oudste stadskerk werd gebouwd. Deze aan Sint Jan-Baptist, patroon van de hermieten, en aan Sint-Baafs gewijde kerk lag aan de rand van een reeds bestaande agglomeratie en zal in de IXe-Xe eeuw het centrum van een handelsnederzetting op de westelijke Scheldeoever worden.

\begin{abstract}
The initial ambition of this study was to try and locate the situation of the cell built around the middle of the VIIth century for Bavon, a recluse, by the driars of the community founded little time before by Saint Amand at the junction of the Lys and Escaut rivers in Gand. The author situates this cell on the location of the present Saint-Bavon's cathedral, where was erected, according to the author's hypotesis, before 804 Gand's oldest urban church. Named after Saint John the Baptist, patron saint of the hermits and after Saint Bavon, this church situated at the limit of a pre-existing urban area, has become in the IXth centuries the centre of a trading area on the left bank of the Escaut.
\end{abstract}

\title{
Résumé
}

Le premier but de cette étude a été de déterminer l'emplacement de la cellule construite vers le milieu du VIle siècle pour le reclus Bavon par les frères de la communauté fondée quelque temps auparavant par saint Amand au confluent de la Lys et de l'Escaut à Gand. L'auteur situe cette cellule à l'endroit de l'actuelle cathédrale Saint-Bavon, où fut construite, selon lui, avant 804 la plus ancienne église urbaine de Gand. Placée sous le vocable de saint Jean-Baptiste, patron des ermites, et de saint Bavon, cette église, située à la lisière d'une agglomération préexistante, est devenue au IXe-Xe siècle le centre d'une agglomération marchande sur la rive gauche de l'Escaut. 


\section{Saint Bavon et les origines de Gand}

La présente communication n'est que le résumé des arguments d'une hypothèse sur les origines de Gand que j'ai commencé à développer en 1971-1972 et qui depuis lors s'est enrichie de nouveaux éléments, qui n'ont pas encore été tous vérifiés ${ }^{1}$.

Le dernier en date de ceux-ci est dû à feu Dom Nicolas Huyghebaert qui peu de temps avant sa mort avait émis l'hypothèse que l'actuelle cathédrale Saint-Bavon de Gand, qui avant d'être élevée à ce rang au XVI ${ }^{e}$ siècle comme siège du nouvel évêché de Gand, fut, avec le titre de Saint-Jean, la principale église de la ville, aurait comme origine la cellule où saint Bavon, disciple de saint Amand, semble avoir passé comme reclus les derniers mois de sa vie, vers ou peu après le milieu du VIIe siècle $^{2}$. Autour d'un oratoire bâti sur le lieu de cette cellule, où saint Bavon était mort ct cntcrré une première fois et qui était dédié tout naturellement à saint Jean-Baptiste, patron des ermites, se serait développé le premier noyau de la ville de Gand, que nous avions en 1971/1972 précisément cherché à cet endroit, sur base, il est important de le souligner, d'autres arguments.

Adriaan Verhulst : professeur à l'Université de Gand, Blandijnberg 2, B 9000 Gand.

1. - A. Verhulst, "Die Frühgeschichte der Stadt Gent ", Festschrift E. Ennen, Bonn 1972, pp. 108-137; ID., "De vroegste geschiedenis en het ontstaan van de stad Gent", Handelingen van de Maatschappij voor Geschiedenis en Oudheidkunde de Gent, XXVI, 1972, pp. 5-39 (version néerlandaise de l'article précédent); ID., " Neue Ansichten über die Entstehung der flämischen Städte am Beispiel von Gent und Antwerpen ", Niederlande und Nordwestdeutschland. Franz Petri zum 80. Geburtstag, éd. W. Енвrecht - H. Schilling, Cologne-Vienne, 1983, pp. 1-17; ID., “Kritische Studie over de oorkonde van Lodewijk IV van Overzee, koning van Frankrijk, voor de Sint-Pietersabdij te Gent (20 augustus 950)", Bulletin de la Commission royaled'histoire, CL, 1984, pp. 272-327 ; ID., "Leie en Schelde als grens in het portus te Gent tijdens de $x^{e}$ eeuw ", Feestbundel Maurits Gysseling (Naamkunde 17), 1985, pp. 407-419.

2. - N. Huyghebaert, "Quelques chartes épiscopales fausses pour Saint-Pierre au MontBlandin à Gand forgées aux XII et XIII' siècles ", Bull. Comm. royale d'histoire, CXLVIII, 1982, pp. 6163.

REVUE DU NORD - TOME LXVIII - N²69 - AVRIL-JUIN 1986 - PP. 455 À 470 


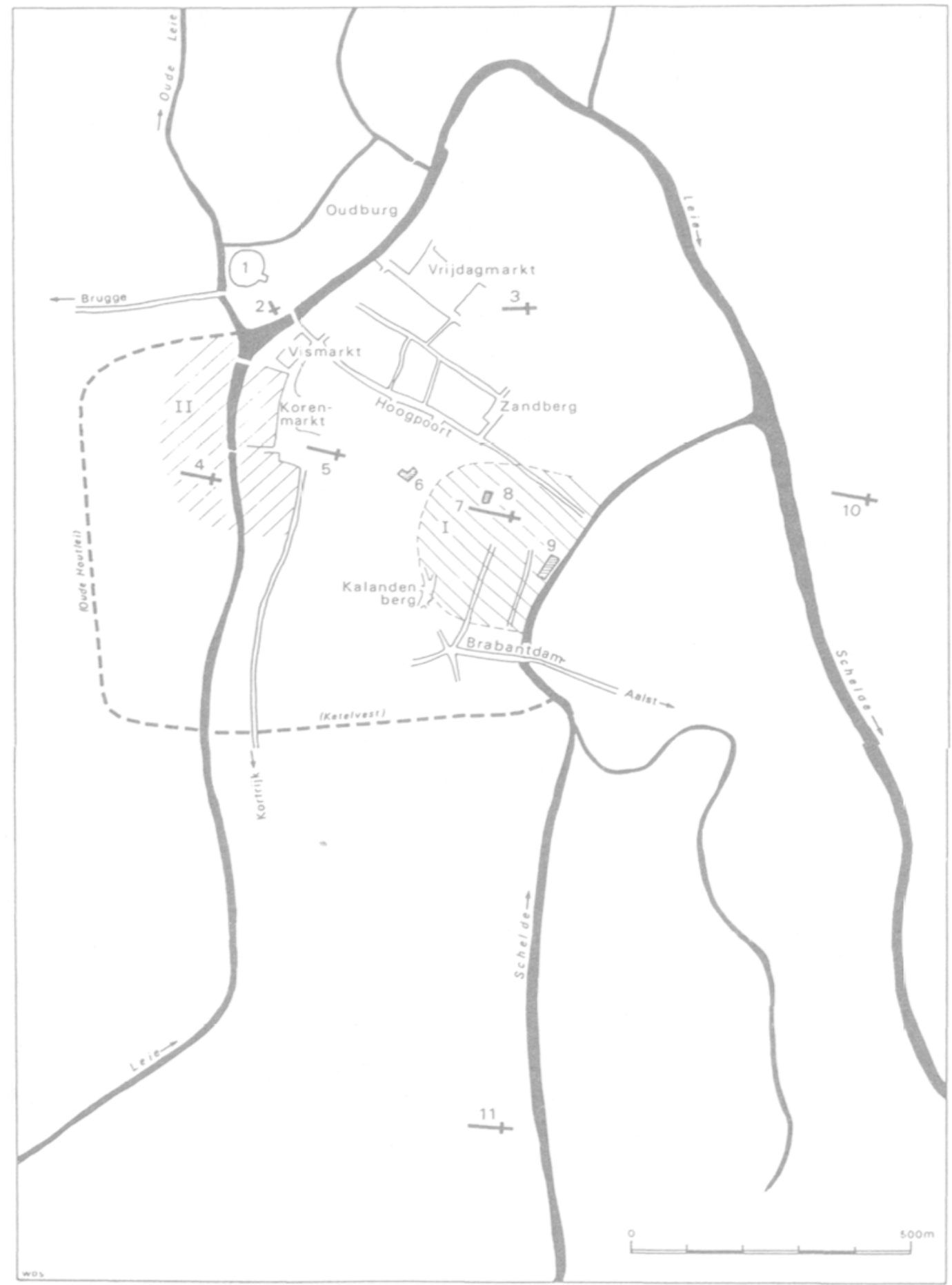

I : Portus du IXe siècle

II : Portus du $\mathrm{X}^{\mathrm{e}}$ siècle

1 : Novum castellum du $\mathrm{X}^{\mathrm{e}}$ siècle (actuel "Château des Comtes")

2 : Eglise castrale Sainte-Pharailde

3 : Eglise Saint-Jacques (XII siècle)

4: Eglise Saint-Michel (fin $X I^{e}$ siècle)

5 : Eglise Saint-Nicolas (fin $\mathrm{XI}^{e}$-début XII ${ }^{e}$ siècle)

6: Beffroi (XIVe siècle)

7 : Eglise Saint-Jean (fin VIII -début IXe siècle)

8 : "Engienhuus " (dépôt des milices urbaines)

9 : "Château de Gérard le Diable " (donjon et demeure primitive du châtelain de Gand ?)

10 : Abbaye de Saint-Bavon

11 : Abbaye de Saint-Pierre-au-Mont-Blandin 
Auparavant on avait, tel Pirenne et ses élèves ${ }^{3}$, cherché l'origine de la ville au Xe siècle au pied de l'actuel Château des comtes, aux bords de la Lys, de l'autre côté de la colline qui sépare ce fleuve de l'Escaut et sur le versant sud-est de laquelle se trouve, au bord de l'Escaut, l'actuelle cathédrale ${ }^{4}$. Ensuite F. Blockmans avait cherché un mystérieux et plus ancien premier portus Ganda, cité vers 865 dans le Martyrologium Usuardi, aux portes mêmes de l'abbaye de Saint-Bavon, au confluent de la Lys et de l'Escaut. Il aurait subi le même sort que l'abbaye, détruite en 879/880 lorsque les Normands y installèrent leur camp d'hiver ${ }^{5}$. Dans les années cinquante le toponymiste Gysseling lança le premier, uniquement sur base de la toponymie urbaine, l'hypothèse que la ville se serait développée à partir d'un village à caractère agricole situé autour du Zandberg, au point le plus élevé de la colline à laquelle ce nom fait allusion, tout près de la cathédrale ${ }^{6}$. Comme cette hypothèse n'était pas rejetée par le meilleur spécialiste de l'histoire de Gand de l'époque, feu notre maître Hans Van Werveke, qui y trouvait surtout une explication à la distance relativement grande qui sépare le portus du $\mathrm{X}^{\mathrm{e}}$ siècle situé aux bords de la Lys, de la plus ancienne église urbaine, située aux bords de l'Escaut, de l'autre côté de la colline séparant les deux fleuves ${ }^{7}$, nous avons depuis lors cherché des arguments historiques, basés sur des sources écrites, en faveur de la localisation proposée par Gysseling. La source principale à cet égard est la Vita Bavonis, écrite vers 825 à l'abbaye de Saint-Bavon, sous l'abbatiat de l'illustre Eginhard, biographe de Charlemagne, en pleine renaissance carolingienne. Conservée dans un manuscrit du $\mathrm{X}^{\mathrm{e}}$ siècle, qui semble transmettre fidèlement le texte original, cette source a aiguisé notre réflexion ${ }^{8}$.

Elle nous apprend que Bavon était un noble originaire de la Hesbaye, donc de la région de Tongres-Maastricht dont l'Aquitain Amand, le principal évangélisateur de la Belgique au VII siècle et notamment fon-

3. - H. Pirenne, "Les villes flamandes avant le XiI" siècle ", Annales de l'Est et du Nord, 1, 1905, pp. 9-32 (réimpression dans ID., Les villes et les institutions urbaines, I, Paris, 1939, pp. 123-141) ; ID., "Coup d'oeil sur l'histoire de Gand ", Gand, Guide illustré, Gand, 1905 (réimpression dans In., Les villes et les institutions urbaines, 11, pp. 141-149); F.-L. Ganshof, Etude sur le développement des villes entre Loire et Rhin au Moyen Age, Paris, 1943 ; H. Van Werveke, Gand. Esquisse d'histoire sociale, Bruxelles, 1946.

4. - Voir notre esquisse, p. 456

5. - F. Blockmans, "De twee opvolgende Gentsche "portus" ", Handelingen van de Société d'Emulation te Brugge, LXXXII, 1939, pp. 52-83; version française sous le titre "Les deux portus successifs de Gand ", Revue du Nord, 26, 1943, pp. 5-20.

6. - M. Gysseling, Gent's vroegste geschiedenis in de spiegel van zijn plaatsnamen, Anvers-BruxellesGand, 1954.

7. - H. VAN WERVEKE, "Opgravingen en navorsingen in verband met de oudste geschiedenis van de stad Gent ", Bijdragen voor de Geschiedenis der Nederlanden, IX, 1954, pp. 33-37.

8. - Ed. B. Krusch, M.G.H., SS. rer. Merov., IV, Hanovre-Leipzig, 1902, pp. 527-546. Voyez sur cette source, hormis l'introduction excellente et toujours valable de B. Krusch à son édition : $L$. VAN DER ESSEN, Etude critique et littéraire sur les vitae des saints mérovingiens de l'ancienne Belgique, LouvainParis, 1907, pp. 351-354; M. Coens, "Saint Bavon était-il évêque?", Analecta Bollandiana, LXIII, 1945, pp. 220-241; R. Podevyn, Bavo, Bruges-Bruxelles, 1945 ; A. Verhulst, "Over de stichting en de vroegste geschiedenis van de Sint-Pieters-en de Sint-Baafsabdijen te Gent ", Handelingen van de Maatschappij voor Geschiedenis en Oudheidkunde ie Gent, VII, 1953, pp. 1-51. Voyez aussi les études sur Bavon citées à la note suivante. 
dateur de l'abbaye Saint-Bavon de Gand, occupait dans les années 647650 le siège épiscopal ${ }^{9}$. Après y avoir fait la connaissance d'Amand, Bavon s'était converti et était ensuite, vers 650 donc, venu à Gand. Il avait, en effet, demandé au saint évêque d'être initié à la vie religieuse et avait visité dans ce but plusieurs fondations de saint Amand pour apprendre à connaître la vie monastique. Bavon s'était enfin établi dans la fondation gantoise du grand évangélisateur, au confluent de l'Escaut et de la Lys, mais seulement pour y attendre la construction d'une cellule où il allait mener désormais une vie de reclus. A sa mort, survenue après quelques mois, il fut enterré une première fois, apparemment dans sa cellule, bien que la Vita Bavonis ne le précise pas, puis, nous dit la même source dans un passage tout à la fin de l'œuvre, dans l'abbaye même ${ }^{10}$. Celle-ci fut originellement dédiée à saint Pierre" ${ }^{11}$, mais à partir du deuxième quart du $\mathrm{IX}^{e}$ siècle, elle allait être appelée communément Saint-Bavon ${ }^{12}$ sans que l'église abbatiale ait jamais été dédiée à ce saint, vraisemblablement parce qu'on y avait construit pour recevoir ses restes une riche sépulture, dont la première mention remonte au début du IX ${ }^{e}$ siècle ${ }^{13}$. Le nom de Saint-Bavon surplanta progressivement celui de Ganda, emprunté au nom du site de l'abbaye, que l'on continuait cependant à utiliser encore pendant tout le $\mathrm{IX}^{\mathrm{e}}$ siècle, principalement dans les actes officiels ${ }^{14}$.

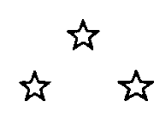

9. - Le travail le plus récent sur saint Bavon et saint Amand est : M. WERNER, Der Lütticher Raum in frühkarolingischer Zeit, Göttingen 1980, pp. 59-72 et pp. 231-236. Parmi les très nombreuses études il faut citer plus particulièrement, à côté de celles citées à la note précédente : M. VAN UYTFanghe, "Bavo ", Lexikon des Mittelalters, I, Munich-Zürich, 1980, col. 1696; ID., "La vallée de l'Escaut et de ses affluents à l'époque mérovingienne : le témoignage des textes", dans A. VAN Doorselaer (éd.), De Merovingische Beschaving in de Scheldevallei, Courtrai 1981, pp. 45-52; A. Verhulst, De SintBaafsabdij te Gent en haar grondbezit (VII-XIV*eeuw), Bruxelles, 1958, pp. 3-43; Lexikon für Theologie, und Kirche, II, Fribourg, 1958, col. 76.

10. - M.G.H., SSRM, IV, p. 545 , ch. 14-15 et ch. 16.

11. - Voir le diplôme de Louis le Pieux de 819 , cité ci-après, note 13.

12. - Voir en dernier lieu WerNer, op. cit., p. 59, n. 2 et surtout Verhulst, De Sint-Baafsabdij, p. 26. C'est dans la Translatio ss. Marcellini et Petri écrite par Eginhard, éd. G. WAIrz, M.G.H. SS, Xv, p. 260, qu'est employée pour la première fois l'expression monasterio Sancti Bavonis. Toutefois l'inventaire du trésor et du domaine de l'abbaye, cité à la note suivante et que nous datons entre 800 et 810/814, nomme systématiquement comme propriétaire des biens sanctus Babo.

13. - Diplôme original de l'empereur Louis le Pieux du 13 avril 819 , éd. M. Grsseling - A.C.F. Косн, Diplomata belgica ante annum 1100 scripta, 1950, $\mathrm{n}^{\circ} 132$ : ex monasterio quod dicitur Ganda, quod est situm in pago Bracbatinse, constructum in honore sancti Petri principis apostolorum, ubi etiam sanctus Bavo confessor Christi corpore requiescit. Le tombeau de saint Bavon dans l'abbaye est mentionné pour la première fois dans un inventaire du trésor et du domaine de l'abbaye, conservé en palimpseste, que nous avons publié et daté entre 800 et 810/814: A. Verhulst, "Das Besitzverzeichnis der Genter SanktBavo-Abtei von ca. 800 (Clm 6333)", Frühmittelalterliche Studien, 5, 1971, pp. 193-234.

14. - Diplôme original de Charles le Chauve du 11 octobre 864, éd. G. 'I'essier, Recueil des acles de Charles II le Chauve, II, Paris, 1952, n²74, p. 116 : monasterii sancti Petri et sancti Bavonis quod vocatur Ganth. 
Le problème principal, surtout par rapport aux hypothèses concernant la localisation du premier noyau urbain de Gand, tourne autour du lieu précis où fut construite vers le milieu du VII e siècle la cellule de saint Bavon.

Diverses opinions avaient déjà été avancées à ce sujet lorsque, dans mon étude de $1972^{15}$, je défendis la thèse que ce devait être aux bords de l'Escaut, en amont de l'abbaye et non loin de celle-ci, donc vraisemblablement sur la colline où se trouve l'actuelle cathédrale Saint-Bavon et où est situé également le Zandberg.

Dans la Vita Bavonis, en effet, nous voyons les "fratres" quitter l'abbaye pour construire la cellulc ${ }^{16}$. A la suite d'une péripétie en relation avec cette construction, située par notre source du IX ${ }^{e}$ siècle aux abords immédiats du vicus de l'endroit, un des "fratres" meurt. On transporte son corps à l'abbaye pour l'y enterrer, mais où demeure aussi saint Bavon qui va le ressusciter miraculeusement. Or, ce transport se fait non pas par terre, mais par eau, puisqu'on met le corps du défunt dans un petit bateau qui descend ensuite le fleuve jusqu'à l'abbaye.

L'hypothèse d'une localisation de la cellule d'ermite de saint Bavon sur la colline près de l'Escaut où se trouve l'actuelle cathédrale gantoise et peut-être, comme nous essayerons de le démontrer plus loin, à l'endroit même de cette église, est confirmée par une source indépendante de la Vita Bavonis, bien que postérieure d'environ un siècle et demi à celle-ci, à savoir les Gesta episcoporum Leodiensium de Hériger de Lobbes, qui datent d'avant $980^{17}$. Déjà Bruno Krusch ${ }^{18}$ y avait relevé, dans un chapitre où l'auteur parle de saint Amand, prédécesseur des évêques de Liège, un court passage où Hériger communique un renseignement concernant ses relations avec Bavon, qui ne se trouve point dans la Vita carolingienne de celui-ci, ni dans la Vita Amandi du début du VIIIe siècle, qui elle ne mentionne même pas Bavon, bien que Hériger ait utilisé ces deux sources. Le renseignement nous apprend que saint Amand enferma le pénitent Bavon comme reclus dans une cellule construite "au delà du fleuve" (trans fluvium) ${ }^{19}$. Puisque Hériger est considéré comme un auteur

15. - Citée à la note 1 ("Die Frühgeschichte der Stadt Gent ") où sont citées les hypothèses antérieures concernant la localisation de la cellule, principalement celle de M. Coens, art. cit., p. 230231, qui se prononça en faveur d'une localisation dans l'abbaye même, sur base d'une interprétation différente d'un passage de la Vita Bavonis que nous commentons ci-après. Voir ci-après note 16.

16. - M.G.H., SS. rer. merny., IV, pp. 539-540. Coens, art. cit., p. 231, estime par contre, "qu'il est plus naturel, d'après le contexte, de croire que ces ouvriers étaient allés quérir, hors du bourg, les matériaux destinés à maçonner l'édicule dans l'enceinte du monastère ». Le passage en question (ch. 7) dit cependant clairement: Die quadam huius temporis fratres ipsius monasterii ad obedientiam sunt egressi, ut cellam viri Dei Bavonis construerent. Laborare igitur ceperunt manibus et aedificium fabricare...

17. - Gesta episcoporum Tungrensium, Traiectensium et Leodiensium, éd. R. KöPKE, M. G.H., SS. VII, Hanovre, 1846, pp. 134-189; réimpression dans J.P. Migne, Patrologia latina, CXXXIX, col. 9581066. Sur cette source: S. BaLAu, Les sources de l'histoire de Liège au Moyen Age. Etude critique, Bruxelles 1903 , pp. 123 suiv.

18. - M.G.H., SS. rer. Merov., v, p. 437, п.5.

19. - Heriger, Gesta, I, c. 35, M.G. H., SS., vi, p. 179 ; Migne, PL. 139, col. 1041 : Monasterium sancti Petri illic aedificavit, sanctum Bavonem ex Haspanio praedonem convertit et trans fluvium facta cellula paenitentem reclusit. 
digne de foi, ce renseignement isolé mais très précis provient vraisemblablement d'une tradition orale originaire de Gand, qui est passée sous silence peut-être délibérément par l'auteur de la Vita Bavonis ${ }^{20}$. Pour interpréter correctement la localisation "trans fluvium " de la cellule, il ne faut pas perdre de vue que, d'après le contexte, où l'auteur parle de l'abbaye de Saint-Bavon ${ }^{21}$, celui-ci s'est placé géographiquement sur le site de l'abbaye. La colline où se trouve l'actuelle cathédrale se situe alors, de ce point de vue, effectivement au delà de l'Escaut.

Le double argument fourni d'une part par la Vita Bavonis, mais de façon délibérément peu explicite, d'autre part par Hériger de Lobbes, permet de considérer comme résolu le problème de la localisation de la cellule de reclus de Bavon, en dépit du fait que des sources postérieures ou bien n'en font pas mention, comme les Miracula Bavonis du milieu ou $\mathrm{du}$ troisième quart du $\mathrm{X}^{\mathrm{e}}$ siècle $^{22}$, et la Vita (secunda) metrica Bavonis (977$980)^{23}$, ou bien situent la cellule à l'intérieur des bâtiments claustraux, comme la Vita (tertia) Bavonis rédigée au début du XII e siècle par l'abbé Thierry de Saint-Trond ${ }^{24}$, ou encore affirment que malgré des transformations architecturales plus récentes, la cellule était encore visible à l'extérieur du chœur de l'église abbatiale, comme le dit le Chronicon Bavonis de Jean de Tilrode, source du XIII ${ }^{e}$ siècle fourmillant de légendes ${ }^{25}$.

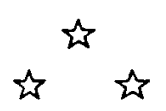

Reste alors le deuxième grand problème, à savoir si un sanctuaire a été construit à l'endroit, au-dessus ou près de cette cellule, avant le milieu du $\mathrm{X}^{\mathrm{e}}$ siècle, puisque ce n'est qu'en 964 qu'est mentionnée pour la première

20. - Sur ses motifs, voyez plus loin p. 464-465.

21. - A vrai dire Hériger désigne l'abbaye fondée par saint Amand comme monasterium sancti Petri (voir ci-devant note 19), ce qui, sous la plume d'un auteur du $x^{e}$ siècle, pourrait signifier l'abbaye de Saint-Pierre au Mont-Blandin. Dans le cadre d'une telle interprétation la réclusion de Bavon trans fluvium devrait alors être localisée à l'abbaye de Saint-Bavon qui, du point de vue de l'abbaye au Mont-Blandin, se trouve, en effet, au delà de l'Escaut. Une telle interprétation se heurte toutefois au récit de la fondation par saint Amand, en l'honneur de saint Pierre, de l'abbaye de Saint-Bavon, tel qu'il est transmis par la Vita Bavonis. Or, non seulement avons-nous démontré jadis (voir notre étude citée note 8) que cette source mérite sur ce point notre confiance, mais Hériger a vraisemblablement suivi à ce propos la tradition de l'abbaye de Saint-Bavon, avec laquelle il entretenait des relations littéraires, comme le démontre le récit de la vie et de la translation vers l'abbaye de Saint-Bavon de saint Landoald et de ses compagnons que Hériger a écrit pour celle-ci : éd. M. Gysseling - A.C.F. Koch, Diplomala belgica, n०138, pp. 234-244 et O. Holder-EgGer, M.G.H., SS., $\mathrm{xV}^{2}$, pp. 599 suiv. Cette œuvre a d'ailleurs joué un rôle, au bénéfice de l'abbaye de Saint-Bavon, dans la dispute de celle-ci avec sa rivale du Mont-Blandin. Voir à ce propos VAN DER ESSEN, Etude critique el littéraire, pp. 357-368.

22. - Livre 1 , ch. 1 , éd. M.G.H., SS., xv², pp. 591-592, où est racontée la réception de Bavon par saint Amand à $\mathrm{G}$ and.

23. - AA. SS., octobre, 1, p. 238, ch. 18 et suiv. Sur cette source: VAN DER EsSEN, Elude critique et littéraire, pp. 354-355.

24. - AA. SS., octobre, 1, pp. 246-252, ch. 18 ct suiv. Sur cette source: VAN DER ESSEN, op. cit., pp. 356-357.

25. - M.G.H., SS., XXV, p. 565. 
fois, dans un diplôme original du roi de France Lothaire V pour l'abbaye Saint-Pierre-au-Mont-Blandin de $\operatorname{Gand}^{26}$, l'église urbaine dédiée à saint Jean-Baptiste, saint Vaast et saint Bavon ${ }^{27}$, que, dans son hypothèse exposée au début de notre article ${ }^{28}$, feu Dom Huyghebaert proposait de considérer comme l'oratoire bâti au plus tard à la fin du VIII ${ }^{e}$ siècle à l'endroit de la cellule de Bavon, construite elle-même, d'après Huyghebaert, sur les ruines d'un fanum romain ${ }^{29}$.

Les origines de cette église sont obscurcies d'une part à cause du mutisme à son égard de la Vita Bavonis du $\mathrm{IX}^{e}$ siècle, écrite après que les restes de saint Bavon eurent été déposés dans le tombeau richement décoré à l'intérieur de l'église abbatiale et peu explicite ou même délibérément obscure en ce qui concerne l'éventuel transfert vers l'abbaye du corps du saint de la cellule où il était mort et enterré une première fois ; d'autre part à cause des falsifications auxquelles a donné lieu aux $\mathrm{X}^{\mathrm{e}}$ et $X^{e}$ siècles, à l'abbaye de Saint-Pierre-au-Mont-Blandin, le fait que cette dernière était devenue propriétaire de l'église Saint-Jean-Baptiste seulement en 964, en dépit des droits moraux et peut-être même juridiques et ecclésiastiques que pouvait faire valoir sur cette église l'abbaye de SaintBavon, grande rivale de sa sœur bénédictine ${ }^{30}$. Ces falsifications avaient eu pour but de faire croire que l'église Saint-Jean-Baptiste avait été construite à l'occasion de la restauration de l'abbaye de Saint-Pierre en 941 942 ainsi qu'à l'initiative de celle-ci et que le titre de saint Jean avait été choisi en l'honneur du premier abbé de Saint-Pierre, appelé Jean ${ }^{31}$. Le but était donc visiblement de faire oublier les antécédents de cette église, que rappellent non seulement le titre de saint Jean-Baptiste, patron des ermites $^{32}$, mais, bien qu'en troisième lieu seulement, celui de saint

26. - Ed. Gysseling-Kосн, Diplomata Belgica, nº6, p. 156 (L. Halphen - F. Lot, Recueil des actes de Lothaire et de Louis $V$, rois de France (954-987), Paris, 1908, n²2, p. 47): In ipso quoque portu (Gandensi) omnes mansioniles cum ecclesia in eo sita. A propos de ce passage du diplôme, voyez VERHULST, "Kritische studie", p. 294 et ID., "Leie en Schelde als grens", pp. 416-418.

27. - Sur ce triple titre, voyez ci-après p. 462.

28. - Voyez plus haut note 2.

29. - La présence de restes romains à cet endroit peut être déduite, selon HuYGHEBAERT, art. cit., p. 61, n.175, du texte même de la Vita Bavonis. Des traces d'un tombeau à incinération de l'époque romaine furent découvertes en 1811 sous une maison près de l'actuelle cathédrale : voyez J. DE BAst, Second supplément au Recueil d'antiquités romaines et gauloises de la Flandre, Gand, 1813, pp. 198199. A propos d'indices archéologiques d'une occupation romaine à divers endroits de la ville actuelle, qui semblent plus nombreux que l'on ne l'ait cru jusqu'à présent, voyez: J. VANDENHOUTE, "Nieuwe perspectieven voor het gallo-romeinse Gent ", dans 45. Congrès de la Fédération des Cercles d'Archéologie et d'Histoire de Belgique. Congrès de Comines 1980. Acles 1, Comines, 1980, pp. 154156.

30. - Sur cette rivalité et ses conséquences, notamment pour les sources diplomatiques de l'abbaye de Saint-Pierre au Mont-Blandin, voir en dernier lieu, après les études classiques à ce sujet de O. Holder-Egcer " $\mathrm{Zu}$ den Heiligengeschichten des Genter Sankt-Bavo-klosters", Historische Aufsätze G. Waitz, Hannovre, 1883, pp. 622-655 et de O. Oppermann, Die älteren Urkunden des Klosters Blandinium und die Anfänge der Stadt Gent, I, Utrecht, 1928 : Verhulst, De Sint-Baafsabdij, pp. 58-83; ID., "Kritische Studie ", p. 282-291.

31. - Verhulst, "Kritische Studie ", pp. 282-291.

32. - Huyghebaert, "Quelques chartes épiscopales fausses", p. 62 et note 178. Voir, en outre, sur le titre de saint Jean Baptiste: M. Beck, Die Patrozinien der ältesten Landkirchen im Archidiakonat Zürichgau, Zürich, 1933, p. 103. Cette dernière référence nous a été aimablement communiquée par G. Berings, licencié en histoire, qui, sur base d'une thèse de licence sur les titres des églises dans les 
Bavon, patron de l'abbaye rivale, titre que l'abbaye du Mont-Blandin apparemment n'avait pu faire disparaître, ce qui démontre bien que sa présence était d'origine plus ancienne ${ }^{33}$.

Cette origine nous la retrouvons peut-être dans un distique composé par le célèbre humaniste carolingien Alcuin comme titulus pour une église dédiée à saint Bavon que personne n'a pu ou osé identifier jusqu'à présent ${ }^{34}$ : "Haec loca sanctificet venerandus Bavo sacerdos / Discipulus vita patris condignus Amandi ". Puisque l'église abbatiale de l'abbaye de Saint-Bavon, malgré l'appellation commune de l'abbaye d'après ce saint à partir du deuxième quart du IX $\mathrm{IX}^{\mathrm{e}}$ siècle, n'a jamais été dédiée qu'à saint Pierre ${ }^{35}$; puisque d'autre part toutes les églises dédiées à saint Bavon que l'on connaît sont de simples églises rurales, la plupart fondées aux IX ${ }^{\mathrm{e}}-\mathrm{X}^{\mathrm{e}}$ siècles par l'abbaye ainsi nommée et dépendantes d'elle ${ }^{36}$; puisqu'enfin il est peu probable que le distique d'Alcuin ait été destiné à une petite église rurale, il est fort bien possible qu'Alcuin ait composé son distique à l'intention de l'église bâtie sur le lieu de la cellule et de la première sépulture du reclus Bavon à une date qui se situe nécessairement avant sa mort en 804 .

Nous nous rendons compte des difficultés qui restent à résoudre pour accepter pleinement cette identification. La principale réside désormais, si l'on admet les interprétations et les argumentations que nous avons développées jusqu'à présent, dans le triple titre de l'église, dédiée, dans l'ordre (d'après une des falsifications de l'abbaye de Saint-Pierre du XI ${ }^{\mathrm{e}}$ siècle qui visiblement n'a rien pu y changer), à saint Jean Baptiste, à saint Vaast et à saint Bavon ${ }^{37}$. Il s'agit plus précisément de savoir pour-

vallées de l'Escaut et de la Lys au Moyen Age, nous signale que le titre de Saint-Jean est très rare parmi les églises dépendant de l'abbaye de Saint-Pierre au Mont-Blandin.

33. - VerhULST, "Frühgeschichte", p. 122. Voir ci après p. 463.

34. - Alcuini Carmina, CVI, hI, éd. E. Dümmler, M.G.H., Poetae latini, I, Berlin, 1881, p. 333. Sur ce texte: Krusch, M.G.H., SS. rer. merov., IV, p. 527 ; Coens, "S. Bavon était-il évêque? ", p. 236 ; Werner, Der Lütticher Raum, p. 61, n.11; VAN UytFanghe, art. "Bavo", Lexikon des Mittelalters, col. 1696.

35. - Voir plus haut, notes 11,12 et 13. L'église paroissiale, également située dans l'enceinte de l'abbaye, mais érigée seulement au XI'e siècle, était dédiée au Christ lui-même : voir G. DE CLERCQ, "De kerkelijke instellingen te Gent in verband met de oudste stedelijke geschiedenis ", Nederlandsche Historiebladen, II, 1939, pp. 119-121.

36. - La liste se trouve dans R. Podevyn, Bavo, Bruges-Bruxelles, 1945, p. 79. Voir aussi L. Voet, De brief van abt Othelbold aan gravin Otgiva, Bruxelles, C.R.H., 1949, pp. 142-145.

37. - La dédicace de l'église en l'honneur de ces trois saints est attribuée à Transmar, évêque de Noyon-Tournai, par un faux diplôme de cet évêque de 947 , appelé preceptum, fabriqué vraisemblablement par l'abbé Guichard vers 1035-1036 lors de la composition de son fameux Liber Traditionum, dans lequel il figure (éd. A. FAYEN, Liber Traditionum Sancti Petri Blandiniensis, Gand, 1906, nº62, p. 65). Sur le caractère faux de ce diplôme, reconnu depuis longtemps et la date de sa fabrication: $\mathrm{N}$. HuYgheBaert, Une translation de reliques à Gand en 944, Bruxelles, 1978, pp. LXV, 47 ; ID., "Quelques chartes épiscopales fausses", pp. 3-11 ; Verhulst, "Kritische Studie", pp. 283-285. Si ce faux comporte quelques données vraies, c'est surtout celle du triple titre, provenant peut-être d'une nota dedicationis: voir à ce propos N. Huyghebaert, "Dedicationes Tornacenses (616-1395) ", Horae Tornacenses, Tournai, 1971, pp. 13-14 et ID., "Quelques chartes épiscopales fausses ", p. 11, n. 41 et p. 61, n. 177. Cette donnée concernant la dédicace, dépourvue de date dans le faux diplôme de Transmar de 947 cité ci-dessus, fut reprise dans une deuxième fausse charte de cet évêque de 941 , fabriquée au XII siècle (éd. H. Pirenne, B.C.R.H., v, 1895, pp. 124-126; voir HuYghebaert, loc. cit.) mais dans laquelle, cette fois, le faussaire a inventé une date pour cette dédicace. La place et le rôle de ces deux 
quoi Saint Bavon ne figure qu'en dernier lieu alors que c'est à lui seul qu'Alcuin a destiné son distique. On pourrait supposer que saint Vaast ne s'y est trouvé qu'à partir de ce que l'on doit dorénavant considérer comme une nouvelle consécration de cette église, notamment par l'évêque de Noyon-Tournai Transmar qui, avant de devenir évêque en 937, avait été prévôt de l'abbaye de Saint-Vaast à Arras.

Cette consécration eut vraisemblablement lieu en 947 , à l'occasion de la restauration de l'abbaye de Saint-Bavon que Transmar semble avoir prise à cœur après que le comte de Flandre, quelques années auparavant, avait favorisé uniquement la restauration de l'abbaye rivale de SaintPierre $^{38}$. Mais on peut tout aussi bien admettre que le titre de saint Vaast remonte plus haut dans le temps, puisqu'il fut, comme saint Amand, un ermite aquitain en l'honneur de qui Alcuin composa d'ailleurs une vita ${ }^{39}$. C'est sans aucun doute le cas pour le titre de saint Jean Baptiste, que l'abbaye de Saint-Pierre au Mont-Blandin a dû trouver à la première place lorsqu'en 964 elle devint enfin maître de l'église. Elle a, en effet, essayé au début du XI ${ }^{\mathrm{e}}$ siècle d'expliquer ce titre à l'aide d'une histoire inventée de toutes pièces, comme nous l'avons signalé plus haut ${ }^{40}$. C'est donc qu'elle n'était pour rien dans la primauté du patron des ermites, dont le patronage de l'église gantoise remonte par conséquent plus haut que le $\mathrm{X}^{\mathrm{e}}$ siècle, c'est-à-dire au $\mathrm{IX}^{\mathrm{e}}$ siècle au plus tard. Puisque l'on peut tenir, avec plus de force encore, un raisonnement analogue à propos de la présence de saint Bavon dans le titre d'une église appartenant à l'abbaye du Mont-Blandin, grande rivale de Saint-Bavon, il est très probable que la plus ancienne église gantoise elle-même, avec le triple titre de saint Jean Baptiste, saint Vaast et saint Bavon, existait déjà au IX ${ }^{e}$, voire au VIII $^{e}$ siècle, qu'elle avait été construite en l'honneur du reclus Bavon et à l'endroit de sa cellule, où se trouvait aussi sa première sépulture. Le fait que le distique d'Alcuin ne mentionne que saint Bavon seul n'infirme pas, à notre avis, cette conclusion et, dès lors, la renforce ${ }^{4 !}$.

\footnotetext{
faux dans l'historiographie de la restauration de l'abbaye de Saint-Pierre au Mont-Blandin au $x^{e}$ siècle apparaîtront dans une étude que nous consacrerons bientôt à la chronologie de cette restauration, d'où il ressortira notamment que l'explication des origines de l'église Saint-Jean-Baptiste dont il est question ici, était l'une des préoccupations majeures des faussaires blandiniens du $\mathrm{X}^{e}$ au $\mathrm{XII^{e }}$ siècle. Il faut faire remarquer enfin que dans la grande charte de confirmation de biens pour l'abbaye du Mont-Blandin de 1038 par le roi Henri jer de France et dans l'obtention de laquelle le faussaire Guichard, abbé de Saint-Pierre, a joué un grand rôle (voir à ce propos Koch - GySSELING, Diplomata belgica, pp. 92-93), l'église en question est citée avec le seul titre de saint Jean-Baptiste (aecclesiam quoque in ipso portu quae est in honore sancti lohannis Baptistae) (éd. Gyssel.ing - Koch, Diplomata belgica, $\mathrm{n}^{\circ} 92$, p. 197). L'omission du titre des deux autres saints est significative, à notre avis, dans une charte obtenue à la demande de l'abbé Guichard.

38. - Sur cette consécration que nous considérons comme historiquement vraie, mais qui n'était, à nos yeux, qu'une nouvelle consécration d'une église déjà existante, voir la note précédente, en attendant l'étude plus approfondie que nous y annonçons.

39. - Lexikon für Theologie und Kirche, X, Fribourg, 1965, col. 649; M. Rouche, L'Aquitaine des Wisigoths aux Arabes (418-781), II, Lille, 197i, p. 454.

40. - Voir notes 30 à 33 et ci-dessus note 37.

41. - A l'occasion du colloque saint Géry à Cambrai, le 6 octobre 1984, où nous avons défendu ce point de vue, M. Atsma a fait remarquer que le vrai patron de l'église ne figure pas toujours en premier lieu dans le titre de celle-ci.
} 
Le dernier problème concerne l'interprétation du silence de la Vita Bavonis au sujet de cette église, dont nous venons de démontrer qu'elle existait vraisemblablement au moment de la rédaction de cette source. La difficulté provient principalement de la façon peu claire et peu conséquente dont la vita raconte les derniers moments du reclus, enfermé dans sa cellule.

Après avoir décrit déjà aux chapitres 12 et 13 , à l'aide de beaucoup d'emprunts, notamment au Nouveau Testament, l'état d'âme et les visions du mourant, l'auteur de la vita, au chapitre 14 , devient tout à coup plus concret et plus original lorsqu'il déclare vouloir "insérer " à cet endroit de sa narration comment Bavon est mort ${ }^{42}$. Bavon fait savoir à l'abbé et aux frères de l'abbaye sous l'autorité desquels il a mené sa vie de reclus ${ }^{43}$, qu'il va mourir et donne l'ordre au serviteur qui semble l'avoir assisté tout au long de sa réclusion, d'aller chercher au monastère de Torhout, au sud-ouest de Bruges, le prêtre Domlinus. Celui-ci, à son arrivée, est chargé par Bavon de prendre soin de ses funérailles au sujet desquelles l'hagiographe ne dit d'ailleurs plus rien d'autre, si ce n'est l'envoi par sainte Gertrude de Nivelles, à qui l'esprit de Bavon est apparu, de vêtements pour ensevelir son corps ${ }^{44}$. Au chapitre suivant ( $\left.{ }^{\circ} 15\right)$, au moment même - dit l'auteur - où l'office des funérailles vient de se terminer, déjà un miracle a lieu, bientôt suivi d'un deuxième miracle, cette fois sur le tombeau du saint. L'auteur annonce qu'il ne va pas poursuivre l'énumération des autres miracles, pour ne pas ennuyer le lecteur et finit tout à coup son récit par une phrase dans laquelle il dit que le saint repose avec grand honneur dans le monastère appelé Ganda, situé au lieu dit "Gandavum castrum " 4 .

Cette brusque fin de l'ouvrage, de même que la construction curieuse, avec des intercalations, des derniers chapitres de la Vita Bavonis, le caractère vague des renseignements au sujet des funérailles proprement dites, qui se passent apparemment en dehors de l'abbaye et probablement dans la cellule même, la manière enfin dont l'auteur, après le récit de deux

42. - M.G.H., SS. rerum Merovingicarum, IV, p. 544. Sur ce passage : Coens, "S. Bavon était-il évêque?", pp. 228-229.

43. - Sur les rapports de Bavon avec "son" abbaye, voir Coens, art. cit., pp. 229-230.

44. - M.G.H., SS. rer. Merov., IV, p. 545 : Statimque virgini beatissimae Gertrudi... spiritus ipsius (Bavonis) apparens, iubet sibi vestimenta mitti ad sepeliendum corpus suum; quod et factum est. (ch. 15) Cumque funeris illius officium expleretur...

45. - M.G.H., SS. rer. Merov. , IV, p. 545 : (ch. 16)... ne studiosis lectoribus et ad alia festinantibus fastidium ingerere videamur. Sepultus namque est vir Dei cum magnifico honore in loco qui dicitur Gandavum castrum, cuius nunc cenobium aperle vocatur Ganda. Sur ce passage curieux, l'insistance qu'y met l'auteur, ainsi que sur la signification et l'identification du Gandavum castrum, voir: VerHulsr, "Over de stichting en vroegste geschiedenis" (cité plus haut note 8), pp. 18-23. 
miracles, insiste sur le lieu exact de la sépulture du saint, qu'il revendique expressément pour son abbaye - tous ces éléments traduisent, à notre avis, l'embarras de l'auteur de la Vita Bavonis devant le fait qu'avant la construction du tombeau du saint à l'abbaye de Saint-Bavon, il a été enseveli dans sa cellule même, d'où son corps doit dès lors avoir été transféré vers l'abbaye à un moment donné ${ }^{46}$. Ce moment, d'après ce que nous savons sur l'existence de ce tombeau, doit se situer avant le début du IXe siècle ${ }^{47}$.

Visiblement l'auteur de la Vita Bavonis a délibérément laissé dans l'ombre la cellule de saint Bavon comme lieu de son premier enterrement, afin de réserver toute la gloire au tombeau situé à l'intérieur de l'abbaye et où le culte du saint s'est développé effectivement dans le courant du deuxième quart du $\mathrm{IX}^{\mathrm{e}}$ siècle ${ }^{48}$. Un état de choses analogue peut être observé à Saint-Riquier, Moyenmoutier et ailleurs. Il n'a donc rien d'anormal, au contraire : toutes ces églises ont voulu exploiter à leur profit les miracles produits à l'intervention d'un saint, souvent reclus, enterré d'abord en dehors de l'église et transféré par après vers celle-ci ${ }^{49}$. Les seuls détails plus ou moins concrets au sujet de la cellule que l'auteur de la Vita Bavonis communique sont d'une part l'épisode de sa construction, que nous avons interprété plus haut, et d'autre part la mention de services religieux auxquels Bavon, si l'on peut en croire l'auteur, a pu assister néanmoins depuis sa cellule, malgré la position très inconfortable dans laquelle il s'y trouvait ${ }^{50}$. Nous hésitons toutefois à voir dans ce dernier détail un argument en faveur de l'existence, à l'époque de la rédaction de la Vita Bavonis, d'une chapelle ou église à côté de la cellule et à laquelle celle-ci aurait été adossée ${ }^{51}$. L'existence d'un oratoire près de la cellule, nous croyons en avoir démontré la vraisemblance à partir d'autres arguments.

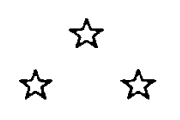

Il convient, pour conclure, de remettre les données au sujet de la cellule de reclus de saint Bavon dans le cadre des origines de la ville, dont le

46. - Aucune donnée écrite ne nous est parvenue au sujet de cette translation : VERHULST, art. cit., p. 19 et note 87.

47. - Voir plus haut note 13.

48. - Voir plus haut notes 12 et 13. Sur le culte de saint Bavon avant la fin du IXe siècle: Coens, "S. Bavon était-il évêque? ", pp. 235-241.

49. - Nous remercions monsieur J. Heuclin, docteur en histoire, auteur d'une thèse de $3^{e}$ cycle sur Ermites et reclus du $V^{*}$ au $V I^{\prime}$ siècle. Aux origines monastiques de la Gaule du Nord, qui nous a signalé ces cas analogues au cours de la discussion qui a suivi notre conférence au colloque saint Géry à Cambrai en octobre 1984 . Voir la discussion ci-après.

50. - M.G.H., SS. rer. Merve., IV, p. 542 (ch. 10).

51. - Voyez plus haut notes 15 et 16 , où nous avons écarté l'hypothèse d'une localisation de la cellule à eôté de l'église abbatiale. 
premier noyau était situé aux abords immédiats de cette cellule et dont la première église remonte vraisemblablement à un sanctuaire construit avant le début du $\mathrm{IX}^{\mathrm{e}}$ siècle à cet endroit. Il faut se poser en premier lieu la question si le culte de saint Bavon à cet endroit a contribué au développement d'une agglomération à caractère urbain. Pour pouvoir répondre à cette question il faudrait connaître avec précision la situation de la cellule de Bavon par rapport à l'emplacement et à l'extension de l'habitat mérovingien dans les environs. Des tessons mérovingiens ont récemment été découverts dans la Gouvernementstraat (au Moyen Age: Hoog- ou Opper-Scheldestraat) immédiatement au sud de l'actuelle cathédrale, dans la Mageleinstraat à environ 150 mètres à l'ouest de cette église, ainsi qu'à une centaine de mètres au sud de cette dernière rue, sur la place dite Kalandenberg, ainsi que dans l'actuelle Kouterdreef. Par contre, des fouilles récentes sur le flanc nord du Zandberg, au nord de la cathédrale, n'ont mis à jour que des traces archéologiques du $\mathrm{XI}^{\mathrm{e}}-\mathrm{XII}^{\mathrm{e}}$ siècle, précédées immédiatement par des restes préhistoriques, sans niveau chronologique intermédiaire ${ }^{52}$. D'après la situation géographique, le dos de colline immédiatement au sud de l'actuelle cathédrale, parallèle dans sa direction sud-nord au cours de l'Escaut, débouchant vers le sud, non loin de l'ancien pont de Brabant et de son moulin à eau, à la grande route vers le Brabant, appelée Brabantdam, croisant à cet endroit l'Escaut, semble le mieux correspondre à la fois à l'emplacement idéal d'une future agglomération marchande et aux trouvailles archéologiques mérovingiennes. L'absence de restes archéologiques importés de cette haute époque, notamment de la céramique du type Badorf, relativement abondante par contre sur le site de l'abbaye de Saint-Bavon, à 500 mètres en aval, ne permet malheureusement pas pour l'instant de soutenir, du point de vue archéologique, l'hypothèse d'une agglomération marchande de l'époque carolingienne à cet endroit.

Peut-être celle-ci s'est-elle développée plus en aval, précisément autour de l'actuelle cathédrale et entre celle-ci et l'Escaut, où se trouvent plusieurs débarcadères à nom en -aard ${ }^{53}$. Notre unique source écrite, la Vita Bavonis, dont le témoignage ne vaut strictement que pour l'époque de sa rédaction, c'est-à-dire vers 825 , situe la cellule de saint Bavon non pas dans, mais à côté d'un vicus préexistant ${ }^{54}$. Cet emplacement peut être interprété éventuellement à la lumière d'une évolution de l'ermite installé à la campagne vers un ermite de type monastique, comme le pense

52. - Nous empruntons ces données à des notices éparses publiées dans la revue Stadsarcheologie, de la ville de Gand, $3,1979, n^{\circ} 2$, pp. $24-35 ; 8,1984, n^{\circ} 2$, pp. $41-42 ; n^{\circ} 3$, p. $56 ; 10,1986, n^{\circ} 1, p$. 36 , ainsi qu'à une étude inédite de $P$. Raveschot, à qui nous devons, comme à sa collègue du Service archéologique de la ville de Gand, M.-C. Laleman, d'autres renseignements précieux concernant les trouvailles archéologiques récentes dans l'ancien centre de la ville.

53. - Verhulst, "Frühgeschichte", p. 126. Toutefois, des fouilles récentes effectuées à cet endroit, n'ont pas fourni d'indications à ce sujet (communication de M.C. Laleman et de P. Raveschot).

54. - M.G.H., SS. rer. Merov., IV, p. 540 (ch. 7). 
J. Heuclin ${ }^{55}$, la transition s'effectuant par une réclusion à mi-chemin de la campagne et de la ville.

Quoi qu'il en soit, nous croyons pouvoir conclure provisoirement et hypothétiquement à l'existence d'un habitat mérovingien au sud de l'actuelle cathédrale, dont le caractère - agricole, administratif ou marchand - ne peut être déterminé pour l'instant. A côté et en dehors de ce noyau habité préexistant, une cellule a été construite pour saint Bavon au milieu du VII ${ }^{e}$ siècle à l'endroit de l'actuelle cathédrale, peut-être sur l'emplacement d'une aire d'inhumation romaine ${ }^{56}$ ou d'autres ruines romaines. Le saint gantois y a été enterré une première fois, un culte s'y est bientôt développé en son honneur, donnant lieu à l'érection d'un sanctuaire placé sous le triple vocable de saint Jean Baptiste, patron des ermites, saint Vaast, ermite lui aussi et saint Bavon. C'est pour cette église qu'Alcuin composa avant 804 son fameux distique, peut-être à l'occasion de sa consécration comme église, située désormais entre le noyau primitif mérovingien d'une part et une nouvelle agglomération, bientôt de caractère marchand, née autour de cette église et entre celle-ci et l'Escaut, d'autre part. Le culte de saint Bavon a très probablement contribué au développement de cette agglomération ${ }^{57}$. L'abbaye voisine de Ganda, dédiée à saint Pierre et sous l'autorité et avec l'aide de laquelle Bavon avait commencé sa vie de reclus, voyant avec envie comment l'église au delà de l'Escaut profitait du culte des restes du saint, fit transporter ceux-ci vers l'abbaye où ils furent mis dans une sépulture magnifique. Comme celle-ci existait déjà au début du $I^{\mathbf{e}}$ siècle, il ne nous semble pas exclu que la consécration d'une nouvelle église au-delà de l'Escaut vers la même époque, ait été l'occasion pour l'abbaye de Ganda, qui n'était peut-être pas étrangère à sa construction, de transférer les restes convoités du saint vers l'abbaye, où se développera bientôt à nouveau son culte, qui fera changer progressivement le nom de celle-ci de Ganda en Saint-Bavon. La Vita Bavonis elle-même a été à la fois le produit et l'instrument de ce culte, essayant de glorifier saint Bavon comme ermite, affirmant avec force la présence de ses restes dans l'abbaye même, tout en restant très vague sur le lieu de sa réclusion et de son premier tombeau.

Adriaan Verhulst

55. - Renseignement aimablement communiqué par $\mathbf{M}$. Heuclin et que l'on trouvera développé dans son travail à paraître, cité plus haut note 49 .

56. - Voir plus haut note 29.

57. - Une preuve en faveur de cette hypothèse serait la foire annuelle qui se tenait jusqu'au début du $\mathrm{XI}^{e}$ siècle à un endroit difficile à déterminer, lors de la fête de saint Bavon, le $1^{\text {er }}$ octobre. bien qu'on estime généralement que les foires du haut Moyen Age, qui se tenaient en dehors des agglomérations marchandes, n'ont pas contribué aux origines de celles-ci : E. ENNEN, Die europäische Stadt des Mittelalters, Göttingen, $1979^{3}$, p. 64 . La thèse inverse a cependant été défendue par $\mathbf{A}$. LOMBARD-JOURDAN, "Du problème de la continuité : y a-t-il une protohistoire urbaine en France?". Annales. Economies-Sociétés-Civilisations, XXV, 1970, pp. 1121-1142 et ID., "Les foires aux origines des villes", Francia, X, 1982, pp. 429-448. Au sujet de la foire de saint Bavon et de sa localisation: VERHulst, "Frühgeschichte", p. 129 et IDEM, "Probleme der Stadtkernforschung in einigen flämischen Städten des frühen und Hochmittelalters" (à paraître dans les actes du colloque Stadtkernfors. chung (1983), dans la série "Städteforschung” de l'Institut für vergleichende Städtegeschichte à Münster). 


\section{Discussion}

Stéphane Lebecq : Monsieur Verhulst a relevé une apparente contradiction entre l'exaltation dans la Vita Bavonis d'un ermite et un contexte qui a été évoqué comme plutôt hostile à une spiritualité érémitique. Il y aurait peut-être là un argument qui renforcerait votre hypothèse. Bavon étant mort, son érémitisme représentait moins de danger à l'époque de Charlemagne ou de Louis le Pieux que de son vivant où il pouvait être considéré comme un marginal refusant de se plier à la règle bénédictine. La contradiction pourrait être résoluc donc par cette focalisation de l'habitat autour de sa cellule. L'ermite n'étant plus ermite que dans un passé lointain.

Adriaan Verhulst : La Vita a eu une certaine difficulté à faire le compromis entre cet érémitisme et la vie monacale. Bavon apparaît non comme un moine mais comme quelqu'un qui est sous l'autorité spirituelle de l'abbé de Saint-Bavon. Il faut tenir compte de la tradition orale: on a beaucoup discuté pour savoir si la Vita Bavonis du IX'e siècle était la première ou s'il y en avait eu une autre datant de l'époque mérovingienne. En réalité personne ne croit plus à cette dernière possibilité mais selon moi, une tradition orale a existé que traduit encore le double enterrement relaté à la fin de la Vita.

Pierre Leman : Quel est l'état de la question archéologique autour de la cathédrale actuelle?

Adriaan Verhulst : Les archéologues ont découvert dans la Gouvernemenstraat, c'est-à-dire sur la même colline que celle où est bâtie la cathédrale, des restes mérovingiens; c'est également dans ce quartier que se trouvent inventoriées depuis la fin du XIX ${ }^{e}$ siècle les plus magnifiques caves romaines d'anciennes maisons patriciennes de Gand. Dom N. Huyghebaert a été plus loin en déduisant d'un passage de la Vita Bavonis que la cellule de Bavon avait été bâtie sur des vestiges romains; de fait on a trouvé au XIXe siècle une tombe romaine à incinération près de l'actuelle cathédrale Saint-Bavon.

Marie-Christine Laleman : Pour le moment on a très peu d'éléments archéologiques pour soutenir l'hypothèse très intéressante qui vient d'être développée sur les origines de Gand et le développement de l'église Saint-Jean. A l'intérieur du demi-cercle nous avons quelques tessons de l'époque mérovingienne. Pour l'époque carolingienne, nous n'avons aucune trace pour le moment dans ce secteur; quant aux maisons patriciennes, la première date du XII ${ }^{e}$ siècle seulement, il y a donc un grand écart avec le haut Moyen Age, et leur densité est aussi importante à l'intérieur qu'à l'extérieur du demi-cercle. 
Michel Rouche : Je voudrais signaler à monsieur Verhulst que le triple patronage de l'église primitive s'explique dans une perspective érémitique. Nous avons saint Jean-Baptiste, saint Vaast et saint Amand qui sont deux ermites aquitains. Ces gens connaissent encore mal la règle de saint Benoît et sont à un stade de spiritualité qui favorise beaucoup plus l'érémitisme que le cénobitisme.

Jean Heuclin : Je voudrais faire remarquer à monsieur Verhulst que la rédaction d'une Vita Bavonis vers 825 exaltant un reclus n'est pas en contradiction avec le mouvement général de l'érémitisme; puisque l'on assiste vers le milieu du $\mathrm{IX}^{\mathrm{e}}$ siècle à une reprise du mouvement après un creux que je situe entre le milieu du VIII ${ }^{e}$ et le milieu du IX ${ }^{e}$ siècle. Cette Vita Bavonis se situant dans les premices de la reprise, sous l'abbatiat d'Eginhard, montrerait que les préceptes élaborés par l'érémitisme antérieurement ont été assimilés par les Carolingiens et servent de substrat à l'édification d'un nouvel univers.

Deuxièmement vous signalez que selon la chronique de Jean de Trelrode du XIII ${ }^{e}$ siècle la cellule se trouvait à côté du chœur de l'abbatiale : il faut voir là l'influence de la Règle de Grimlaïc, il s'agit donc bien d'une reconstruction mentale a posteriori. Par contre il est extrêmement intéressant de voir une cellule transfluvium associée à saint Jean-Baptiste; l'image est très forte, témoignant d'une régénération, un nouveau baptème du pénitent Bavon et l'annonce d'un monde nouveau avec un nouveau Messie. Je me permets d'insister sur ce symbolisme. Par ailleurs on voit aux VII e-VIIIe siècles une évolution de l'ermite installé à la campagne vers un ermite de type monastique. La transition s'effectue par une réclusion à mi-chemin de la campagne et de la ville. C'est le cas de Vodalus reclus à la porte de l'abbaye Sainte-Marie de Soissons, qui entre d'ailleurs en conflit avec l'abbesse ; la cellule d'Amé à Breuil-sur-la-Lys n'est pas dans le monastère mais sur une colline dominant l'agglomération.

Enfin le rite des deux enterrements apparaît assez souvent : une première fois dans la cellule puis au monastère voisin. C'est le cas de saint Riquier. Je crois que le drame pour une abbaye c'est de voir l'ermite mort faire des miracles; les foules arrivent et perturbent la vie religieuse. C'est ce qui se produit à Moyenmoutiers dans les Vosges où Spinule, par les miracles réalisés à son tombeau, attire deux fois plus de monde que le monastère; deux autres ermites Jean et Bénigne réalisent les mêmes exploits. Les moines décidèrent de ramener les corps et de les mélanger dans un même tombeau au monastère, puis firent reboucher les sources d'eau salée proches qui attiraient les pélerins. Je vous signale qu'en outre Moyenmoustiers a une église dédiée à saint Jean-Baptiste. Une situation très similaire à celle de Bavon existe avec Victor ( † 640) près d'Arcy-surAube enterré dans sa cellule et transféré en 837 à l'abbaye de Montieramé. Tout cela me semble confirmer ce que vous venez de nous indiquer. 
Adriaan Verhulst : Je vous remercie beaucoup.

Hartmut Atsma : Je voudrais ajouter une remarque. Il faut se rappeler que dans toutes les sources, chartes, documents épigraphiques et hagiographiques le patronus ne vient pas toujours en premier lieu. Il y a les apôtres et on ne peut dire que celui qui est cité en premier lieu soit vraiment celui qui donne le vocable à l'église ou au monastère. 